\title{
ON THE PROBLEM OF STOCHASTIC EXPERIMENTAL MODAL ANALYSIS BASED ON MULTIPLE-EXCITATION MULTIPLE-RESPONSE DATA, PART I: DISPERSION ANALYSIS OF CONTINUOUS-TIME STRUCTURAL SYSTEMS
}

\author{
J. E. Lee $\dagger$ AND S. D. FAssois \\ Department of Mechanical Engineering and Applied Mechanics, The University of Michigan, \\ Ann Arbor, Michigan 48109-2121, U.S.A.
}

(Received 7 February 1991, and in final form 25 September 1991)

\begin{abstract}
Despite its importance and the undisputable significance of stochastic effects, the problem of multiple-excitation multiple-response experimental modal analysis has thus far been almost exclusively considered within a deterministic framework. In this paper a novel, comprehensive and effective stochastic approach, that, unlike alternative schemes, can operate on vibration displacement, velocity or acceleration data, is introduced. The proposed approach is capable of effectively dealing with noise-corrupted vibration data, while also being characterized by unique features that enable it to overcome major drawbacks of current modal analysis methods and achieve high performance characteristics by employing: (a) proper and mutually compatible force excitation signal type and stochastic model forms, (b) an estimation scheme that circumvents problems such as algorithmic instability, wrong convergence, and high computational complexity, while requiring no initial guess parameter values, (c) effective model structure estimation and model validation procedures, and, (d) appropriate model transformation, reduction and analysis procedures based on a novel dispersion analysis methodology.

This dispersion analysis methodology is a physically meaningful way of assessing the relative importance of the estimated vibrational modes based on their contributions ("dispersions") to the vibration signal energy. The effects of modal cross-correlations are fully accounted for, physical interpretations are provided in both the correlation and spectral domains, and the phenomenon of negative dispersion modes is investigated and physically interpreted. The effectiveness of the proposed approach is finally verified via numerical and laboratory experiments, as well as comparisons with the classical frequency domain method and the deterministic eigensystem realization algorithm (ERA).

The paper is divided into two parts: the proposed dispersion analysis methodology is introduced in the first one; whereas the complete stochastic experimental modal analysis approach is presented in the second [23].
\end{abstract}

\section{INTRODUCTION}

Time-domain experimental modal analysis approaches may be classified as either deterministic or stochastic. Deterministic approaches [1-6] are often simpler and computationally attractive, but they face significant difficulties in dealing with noise-corrupted experimental data. Indeed, it is well-known [7-10] that the quality of modal parameters identified via deterministic methods decreases drastically as the noise-to-signal $(\mathrm{N} / \mathrm{S})$ ratio ceases to be

† Current address: Department of Production Automation Engineering, Ajou University, Suwon, Korea 441-749. 
negligible, and a number of modes may then be completely impossible to estimate. As a consequence, emphasis has been recently placed on stochastic approaches that can effectively account for the presence of noise in experimental data [7-18].

Most of the available stochastic approaches, however, are of the single-output type, and are therefore restricted to one vibration measurement location. This is a rather severe limitation for practical applications where multiple-excitation multiple-response [also referred to as multiple-input multiple-output (MIMO)] methods are in demand because of a number of advantages that they can offer, including significantly reduced total data acquisition and processing time requirements, the use of more consistent data sets, more uniform distribution of the excitation signal energy within the structure, more "consistent" modal parameter estimates due to the larger number of data used, effective separation of multiple or closely coupled modes, and significantly reduced probability of missing vibrational modes.

Despite these potential advantages, very few stochastic MIMO approaches are currently available. Hu et al. [17] discussed an approach based on multi-variate autoregressive moving average (ARMA) models with parameters obtained through the modified YuleWalker estimator. The performance of this estimator is, however, known to vary greatly, and result in significant identification scatter and reduced accuracy [19]. In addition, an unnecessarily high number of parameters is estimated, the noise dynamics are not identified, and a number of issues (such as model structure estimation, estimation of the actual number of structural degrees of freedom/model reduction, appropriate selection of the excitation signal type and the required ARMA forms, as well as model validation) are neglected. The approach proposed by Bonnecase et al. [20] for the case of unobservable force excitation is also based on the modified Yule-Walker estimator (or its overdetermined form) and is of a very similar nature.

Part of the reason for the lack of stochastic MIMO experimental modal analysis approaches is due to difficulties related to the discrete estimation stage (algorithmic instability occurrence, wrong convergence, the need for initial guess parameter values, high computational complexity), inappropriate selection of the triple of model structure, excitation signal type, and discrete-to-continuous model transformation, the lack of an effective model structure estimation procedure, and the lack of a methodology for assessing the relative importance of the estimated vibrational modes (necessary for model analysis, reduction, and the distinction between "structural" and "extraneous" modes). Although most of these issues (except for the last one) were recently addressed and effectively resolved for the special single-output case [18], those results are not directly extendable to the more general MIMO problem.

Indeed, it is a well-known fact that the extension from the single to the multiple-output case is far from being trivial, as stochastic multi-variate models have a structure that is much more complicated than that of their univariate counterparts, and gives rise to deep identifiability questions that necessitate the use of identifiable parametrizations and special model structure estimation techniques [21]. Additional problems are also encountered as parameter estimation in multi-variate models is much more complicated and prone to difficulties related to local extrema/wrong convergence and algorithmic instabilities, so that the availability of good initial parameter values is of critical importance [22]. The computational complexity of multi-variate estimation algorithms is also excessive, to the point that it is often considered as prohibitive for many practical applications.

Regarding the issue of quantitative assessment of the relative importance of a structure's estimated vibrational modes, very little has been done $[8,9,18]$. This is so despite the issue's apparent significance in model analysis, reduction, and the distinction between actual "structural" and "extraneous" vibrational modes in an estimated structural model. 
Such extraneous modes primarily appear in conjunction with deterministic methods and are mainly due to the latter's inability to cope with noise-corrupted data. However, they are encountered in the results of stochastic methods as well (although in drastically reduced numbers); in that case they are due to the dynamics of imperfect instruments and/or statistical errors and estimator inaccuracies. Although some techniques (such as the modal amplitude coherence used in conjunction with the eigensystem realization algorithm [4]) have been suggested for distinguishing structural from extraneous modes in a deterministic setting, those neither address the aforementioned general problem nor work effectively in environments in which the noise is not negligible $[10,18]$.

It is thus the goal of this work to develop an appropriate methodology for the quantitative assessment of the relative importance of a structural system's modes, and also overcome the aforementioned difficulties and develop an effective and realistic stochastic MIMO experimental modal analysis approach.

The problem of quantitative assessment of the relative importance of a structural model's vibrational modes is studied first, and a novel and physically meaningful methodology, referred to as dispersion analysis, that assesses the significance of each mode by determining its contribution to the total vibration signal energy under broadband excitation conditions, is developed. This methodology fully accounts for all cross-correlation effects (second order statistical interactions) among the various modes, and provides a definite and objective answer to the question of mode importance. Physical interpretations are also provided, and the important phenomenon of modes characterized by negative contributions is considered and existence conditions are derived.

The complete stochastic MIMO experimental modal analysis problem is considered next, and a novel and effective approach that, unlike alternative schemes, is capable of operating on either displacement, velocity or acceleration vibration data records, and utilizes the dispersion analysis methodology for model reduction and analysis, is introduced. The performance characteristics of the proposed approach are examined via numerical and laboratory experiments, and comparisons are made with the classical frequency domain method (FDM) and the deterministic eigensystem realization algorithm (ERA) [4].

This paper consists of two parts: the problem of quantitative assessment of the relative importance of a structure's vibrational modes and the proposed dispersion analysis methodology are discussed in part $\mathrm{I}$; whereas the formulation and testing of the MIMO experimental modal analysis approach is presented in part II [23]. The presentation in part I is organized as follows. The basic formulation of the dispersion analysis methodology is outlined in section 2, and the existence of modes characterized by negative contributions is discussed in section 3. Various interpretations of the proposed methodology, with emphasis on negative dispersion modes and their physical significance, are discussed in section 4 , and the conclusions of the study are summarized in section 5 .

\section{ANALYSIS OF DISPERSION FOR CONTINUOUS-TIME STRUCTURAL SYSTEMS}

Consider a general, linear, viscously damped $n$-degree-of-freedom structural system described by the differential equation

$$
\mathbf{M} \cdot \ddot{\mathbf{v}}(t)+\mathbf{C} \cdot \dot{\mathbf{v}}(t)+\mathbf{K} \cdot \mathbf{v}(t)=\mathbf{f}(t),
$$

in which $\mathbf{M}, \mathbf{C}$ and $\mathbf{K}$ represent the real and symmetric $n \times n$ mass, viscous damping and stiffness matrices, respectively, $\mathbf{f}(t)$ the $n$-dimensional force excitation signal, and $\mathrm{v}(t)$ the resulting $n$-dimensional vibration displacement signal. This system is characterized by $n$ vibrational modes, and hence $n$ pairs of complex conjugate eigenvalues of the form

$$
s_{i}, s_{i}^{*}=-\zeta_{i} \omega_{n_{i}} \pm \mathrm{j} \omega_{n_{i}} \sqrt{1-\zeta_{i}^{2}}=-\zeta_{i} \omega_{n_{i}} \pm \mathrm{j} \omega_{d_{i}} \quad(i=1, \ldots, n)
$$


where $\omega_{d_{i}}$ represents the $i$ th damped natural frequency $\omega_{d_{i}} \triangleq \omega_{n_{i}} \sqrt{1-\zeta_{i}^{2}}, \omega_{n_{i}}$ the $i$ th natural frequency, $\zeta_{i}$ the $i$ th damping factor and $\mathrm{j}$ the imaginary unit. Corresponding to these eigenvalues are $n$ pairs of complex conjugate eigenvectors (mode shapes) of the form

$$
\phi_{i}=\left\{\begin{array}{c}
\phi_{1 i} \\
\phi_{2 i} \\
\vdots \\
\phi_{n i}
\end{array}\right\}, \quad \phi_{i}^{*}=\left\{\begin{array}{c}
\phi_{1 i}^{*} \\
\phi_{2 i}^{*} \\
\vdots \\
\phi_{n i}^{*}
\end{array}\right\}, \quad(i=1, \ldots, n) .
$$

The problem of interest here is that of quantitatively assessing the relative importance of each vibrational mode in the system of equation (1), and for its solution a novel and physically meaningful dispersion analysis methodology, according to which the relative importance of a given mode is judged by its contribution to the vibration energy of the structure under broadband (uncorrelated) stochastic excitation conditions, is introduced. The key idea behind this methodology is in the appropriate decomposition of the vibration energy into modal contributions that will be referred to as (modal) dispersions. The excitation signal is selected as uncorrelated stochastic because it is then characterized by a perfectly flat spectrum that warrants that all frequencies are excited at the same level, with no particular weight being assigned to any frequency or frequency range.

The foundation of this proposed methodology may be traced to the notion of dispersion used in conjunction with discrete time series models [24] which, in turn, has its roots in the statistical analysis of variance. The first use of dispersion analysis in structural dynamics was based on approximate discrete-time models and may be found in the papers by Ben Mrad and Fassois [8,9], Fassois et al. [16] and Lee and Fassois [18]. However, these earlier attempts to address the problem suffer from two major drawbacks.

(1) They are all based on discrete-time models and are therefore approximate and subject to errors relating to the fact that actual structural systems are inherently continuous and not discrete time. These errors are known to be functions of various factors, including the excitation signal type $[8,9]$, and are very difficult to assess. Furthermore, the utilization of discrete models does not allow for physical insight and a clear understanding of the role of the global and local modal characteristics in determining the contribution of each vibrational mode.

(2) The frequently occurring phenomenon of negative dispersion modes has yet to be addressed. As a consequence, a clear understanding of the role of such modes and their physical significance is currently lacking.

It is thus the specific objective of this paper to develop a proper and exact dispersion analysis methodology based on continuous-time structural models, explicitly relate the relative contribution of each vibrational mode to physical quantities of interest (such as natural frequencies, damping factors, and mode shapes), and also provide clear physical interpretations of the notion of modal dispersion and the phenomenon of negative dispersion modes.

\subsection{THE NOTION OF MODAL DISPERSION}

The key idea of the proposed methodology is in the proper decomposition of the vibration displacement signal energy and the introduction of the notion of modal dispersion. Broadly speaking, the modal dispersion is the part of the vibration response energy associated with a given mode under broadband stochastic excitation conditions. For a precise definition the vibration signal variance for a general viscously damped structural system needs to be computed and appropriately decomposed. 
Towards this end the solution of the general structural system equation (1) for a given vector excitation $\mathbf{f}(t) \triangleq\left[f_{1}(t) \cdots f_{n}(t)\right]^{\mathrm{T}}$ is expressed in the form [25]

$$
\mathbf{v}(t)=\sum_{i=1}^{n}\left[\phi_{i} \sum_{k=1}^{n}\left(\frac{\phi_{k i}}{a_{i}} \int_{-\infty}^{t} h_{i}(t-\tau) f_{k}(\tau) \mathrm{d} \tau\right)+\phi_{i}^{*} \sum_{k=1}^{n}\left(\frac{\phi_{k i}^{*}}{a_{i}^{*}} \int_{-\infty}^{t} h_{i}^{*}(t-\tau) f_{k}(\tau) \mathrm{d} \tau\right)\right],
$$

with

$$
\begin{aligned}
& h_{i}(t) \triangleq \mathrm{e}^{s_{i} t}=\mathrm{e}^{-\zeta_{t} \omega_{n_{i}}\left(\cos \omega_{d_{i}} t+\mathrm{j} \sin \omega_{d_{l}} t\right),} \\
& h_{i}^{*}(t) \triangleq \mathrm{e}^{s_{i t} t}=\mathrm{e}^{-\zeta_{i} \omega_{n_{i}}\left(\cos \omega_{d_{i}} t-\mathrm{j} \sin \omega_{d_{l}} t\right),}
\end{aligned}
$$

and with the constants $a_{i}$ and $a_{i}^{*}$ (which are the coefficients of the derivative term in the $i$ th uncoupled state equation using normal co-ordinates) given as

$$
\begin{gathered}
a_{i} \triangleq 2 s_{i} \cdot \phi_{i}^{\mathrm{T}} \mathbf{M} \phi_{i}+\phi_{i}^{\mathrm{T}} \mathbf{C} \phi_{i}, \\
a_{i}^{*} \triangleq 2 s_{i}^{*} \cdot \phi_{i}^{* \mathrm{~T}} \mathbf{M} \phi_{i}^{*}+\phi_{i}^{* \mathrm{~T}} \mathbf{C} \phi_{i}^{*} .
\end{gathered}
$$

The contribution of each vibrational mode will be defined separately for each one of the system's transfer functions. As a consequence, and without any loss of generality, the $m l$ th transfer function relating the force $f_{l}(t)$ to the vibration displacement response $v_{m}(t)$ will be considered. Based on equation (4), the autocorrelation function of the vibration signal $\left\{v_{m}(t)\right\}$ may be computed as

$$
\begin{aligned}
R_{v_{m}}(\tau)= & \mathrm{E}\left[v_{m n}(t) v_{m}(t+\tau)\right] \\
= & \sum_{i=1}^{n} \sum_{k=1}^{n} \mathrm{E}\left[\left(\frac{\phi_{m i} \phi_{l i}}{a_{i}} \int_{0}^{\infty} f_{l}\left(t-\tau_{1}\right) h_{i}\left(\tau_{1}\right) \mathrm{d} \tau_{1}+\frac{\phi_{m i}^{*} \phi_{l i}^{*}}{a_{i}^{*}} \int_{0}^{\infty} f_{l}\left(t-\tau_{1}\right) h_{i}^{*}\left(\tau_{1}\right) \mathrm{d} \tau_{1}\right)\right. \\
& \left.\times\left(\frac{\phi_{m k} \phi_{l k}}{a_{k}} \int_{0}^{\infty} f_{l}\left(t+\tau-\tau_{2}\right) h_{k}\left(\tau_{2}\right) \mathrm{d} \tau_{2}+\frac{\phi_{m k}^{*} \phi_{l k}^{*}}{a_{k}^{*}} \int_{0}^{\infty} f_{l}\left(t+\tau-\tau_{2}\right) h_{k}^{*}\left(\tau_{2}\right) \mathrm{d} \tau_{2}\right)\right],
\end{aligned}
$$

where $\mathrm{E}[\cdot]$ denotes the statistical expectation operator. The first term in this expression may be rewritten as

$$
\begin{aligned}
1 \text { st term } & =\mathrm{E}\left[\left(\frac{\phi_{m i} \phi_{l i}}{a_{i}} \int_{0}^{\infty} f_{l}\left(t-\tau_{1}\right) h_{i}\left(\tau_{1}\right) \mathrm{d} \tau_{1}\right)\left(\frac{\phi_{m k} \phi_{l k}}{a_{k}} \int_{0}^{\infty} f_{l}\left(t+\tau-\tau_{2}\right) h_{k}\left(\tau_{2}\right) \mathrm{d} \tau_{2}\right)\right] \\
& =\frac{\phi_{m i} \phi_{l i} \phi_{l n k} \phi_{l k}}{a_{i} a_{k}} \int_{0}^{\infty} \int_{0}^{\infty} \mathrm{E}\left[f_{l}\left(t-\tau_{1}\right) f_{l}\left(t+\tau-\tau_{2}\right)\right] h_{i}\left(\tau_{1}\right) h_{k}\left(\tau_{2}\right) \mathrm{d} \tau_{1} \mathrm{~d} \tau_{2} \\
& =\frac{\phi_{m i} \phi_{l i} \phi_{l m k} \phi_{l k}}{a_{i} a_{k}} \int_{0}^{\infty} \int_{0}^{\infty} R_{f}\left(\tau+\tau_{1}-\tau_{2}\right) h_{i}\left(\tau_{1}\right) h_{k}\left(\tau_{2}\right) \mathrm{d} \tau_{1} \mathrm{~d} \tau_{2},
\end{aligned}
$$

with $R_{f}(\cdot)$ representing the autocorrelation function of the force excitation signal $\left\{f_{l}(t)\right\}$. In the case of uncorrelated excitation $R_{f}(\cdot)$ is of the form

$$
R_{f}\left(\tau+\tau_{1}-\tau_{2}\right)=R_{f_{0}} \delta\left(\tau+\tau_{1}-\tau_{2}\right)
$$

where $\delta(\cdot)$ represents the Dirac's delta function, and $R_{f_{0}}$ its intensity. The substitution of equation (11) into equation (10) then yields, for $\tau \geqslant 0$, 


$$
\begin{aligned}
\text { 1st term } & =R_{f_{0}} \frac{\phi_{m i} \phi_{l i} \phi_{l n k} \phi_{l k}}{a_{i} a_{k}} \int_{0}^{\infty} h_{i}\left(\tau_{1}\right) h_{k}\left(\tau+\tau_{1}\right) \mathrm{d} \tau_{1} \\
& =\mathrm{e}^{-\zeta_{k} \omega_{n_{k}}{ }^{\tau}}\left(\cos \omega_{d_{k}} \tau+\mathrm{j} \sin \omega_{d_{k}} \tau\right)\left[R_{f_{0}} \frac{\phi_{m i} \phi_{l i} \phi_{m k} \phi_{l k}}{a_{i} a_{k}} \int_{0}^{\infty} h_{i}\left(\tau_{1}\right) h_{k}\left(\tau_{1}\right) \mathrm{d} \tau_{1}\right]
\end{aligned}
$$

By similarly computing the remaining three product terms within the square bracket of equation (9), the autocorrelation function $R_{v_{m}}(\tau)$ may be finally expressed in the decomposition form

$$
R_{\nu_{m}}(\tau) \triangleq \sum_{k=1}^{n} D_{k}(\tau) \triangleq \sum_{k=1}^{n}\left[\exp \left(-\zeta_{k} \omega_{n_{k}} \tau\right)\left(\eta_{1}^{(k)} \cos \omega_{d_{k}} \tau+\eta_{2}^{(k)} \sin \omega_{d_{k}} \tau\right)\right]
$$

with $D_{k}(\tau)$ obviously defined and

$$
\begin{aligned}
\eta_{1}^{(k)}= & R_{f_{0}} \sum_{i=1}^{n}\left(\frac{\phi_{m i} \phi_{l i} \phi_{m k} \phi_{l k}}{a_{i} a_{k}} \int_{0}^{\infty} h_{i}(t) h_{k}(t) \mathrm{d} t+\frac{\phi_{m i} \phi_{l i} \phi_{m k}^{*} \phi_{l k}^{*}}{a_{i} a_{k}^{*}} \int_{0}^{\infty} h_{i}(t) h_{k}^{*}(t) \mathrm{d} t\right. \\
& \left.+\frac{\phi_{m i}^{*} \phi_{l i}^{*} \phi_{m k} \phi_{l k}}{a_{i}^{*} a_{k}} \int_{0}^{\infty} h_{i}^{*}(t) h_{k}(t) \mathrm{d} t+\frac{\phi_{m i}^{*} \phi_{l i}^{*} \phi_{m k}^{*} \phi_{l k}^{*}}{a_{i}^{*} a_{k}^{*}} \int_{0}^{\infty} h_{i}^{*}(t) h_{k}^{*}(t) \mathrm{d} t\right), \\
\eta_{2}^{(k)}= & \mathrm{j} R_{f_{0}} \sum_{i=1}^{n}\left(\frac{\phi_{m i} \phi_{l i} \phi_{m k} \phi_{l k}}{a_{i} a_{k}} \int_{0}^{\infty} h_{i}(t) h_{k}(t) \mathrm{d} t-\frac{\phi_{m i} \phi_{l i} \phi_{m k}^{*} \phi_{k}^{*}}{a_{i} a_{k}^{*}} \int_{0}^{\infty} h_{i}(t) h_{k}^{*}(t) \mathrm{d} t\right. \\
& \left.+\frac{\phi_{m i}^{*} \phi_{l i}^{*} \phi_{m k} \phi_{l k}}{a_{i}^{*} a_{k}} \int_{0}^{\infty} h_{i}^{*}(t) h_{k}(t) \mathrm{d} t-\frac{\phi_{m i}^{*} \phi_{l i}^{*} \phi_{m k}^{*} \phi_{l k}^{*}}{a_{i}^{*} a_{k}^{*}} \int_{0}^{\infty} h_{i}^{*}(t) h_{k}^{*}(t) \mathrm{d} t\right) .
\end{aligned}
$$

Observe that both $\eta_{1}^{(k)}$ and $\eta_{2}^{(k)}(k=1,2, \ldots, n)$ are real-valued since their first and fourth, as well as their second and third, terms are complex conjugate.

From equation (13) it is evident that the autocorrelation function $R_{v_{m}}(\tau)$ has been decomposed into $n$ decaying trigonometric terms $D_{k}(\tau)(k=1,2, \ldots, n)$, each one of which is associated with each one of the system's vibrational modes and represents that mode's contribution to $R_{v_{m}}(\tau)$.

The energy associated with the vibration displacement signal $\left\{v_{m}(t)\right\}$ may be then evaluated from equation (13) by setting $\tau=0$

$$
\begin{aligned}
R_{v_{n}}(0)= & \sum_{k=1}^{n}\left[2 R _ { f _ { 0 } } \sum _ { i = 1 } ^ { n } \operatorname { R e } \left(\frac{\phi_{l m i} \phi_{l i} \phi_{m k} \phi_{l k}}{a_{i} a_{k}} \int_{0}^{\infty} h_{i}(t) h_{k}(t) \mathrm{d} t\right.\right. \\
& \left.\left.+\frac{\phi_{m i} \phi_{l i} \phi_{m k}^{*} \phi_{l k}^{*}}{a_{i} a_{k}^{*}} \int_{0}^{\infty} h_{i}(t) h_{k}^{*}(t) \mathrm{d} t\right)\right]
\end{aligned}
$$

with $\operatorname{Re}(\cdot)$ denoting the real part of the indicated quantity. Since

$$
\begin{aligned}
\int_{0}^{\infty} h_{i}(t) h_{k}(t) \mathrm{d} t & =\int_{0}^{\infty} \mathrm{e}^{-\left(\zeta_{i} \omega_{n_{i}}+\zeta_{k} \omega_{n_{k}}\right) t}\left[\cos \left(\omega_{d_{i}}+\omega_{d_{k}}\right) t+\mathrm{j} \sin \left(\omega_{d_{i}}+\omega_{d_{k}}\right) t\right] \mathrm{d} t \\
& =\frac{\left(\zeta_{i} \omega_{n_{i}}+\zeta_{k} \omega_{n_{k}}\right)+\mathrm{j}\left(\omega_{d_{i}}+\omega_{d_{k}}\right)}{\omega_{n_{i}}^{2}+\omega_{n_{k}}^{2}+2 \zeta_{i} \zeta_{k} \omega_{n_{i}} \omega_{n_{k}}+2 \omega_{n_{i}} \omega_{n_{k}} \sqrt{\left(1-\zeta_{i}^{2}\right)\left(1-\zeta_{k}^{2}\right)}} \\
& \triangleq \eta_{3}^{(i, k)}
\end{aligned}
$$


and

$$
\begin{aligned}
\int_{0}^{\infty} h_{i}(t) h_{k}^{*}(t) \mathrm{d} t= & \frac{\left(\zeta_{i} \omega_{n_{i}}+\zeta_{k} \omega_{n_{k}}\right)+\mathrm{j}\left(\omega_{d_{i}}-\omega_{d_{k}}\right)}{\omega_{n_{i}}^{2}+\omega_{n_{k}}^{2}+2 \zeta_{i} \zeta_{k} \omega_{n_{i}} \omega_{n_{k}}-2 \omega_{n_{i}} \omega_{n_{k}} \sqrt{\left(1-\zeta_{i}^{2}\right)\left(1-\zeta_{k}^{2}\right)}} \\
& \triangleq \eta_{4}^{(i, k)}
\end{aligned}
$$

$R_{v_{m}}(0)$ may be rewritten in the decomposition form

$$
R_{v_{m}}(0) \triangleq \sum_{k=1}^{n} D_{k}(0) \triangleq \sum_{k=1}^{n}\left[2 R_{f_{0}} \sum_{i=1}^{n} \operatorname{Re}\left(\frac{\phi_{m i} \phi_{l i} \phi_{m k} \phi_{l k}}{a_{i} a_{k}} \eta_{3}^{(i, k)}+\frac{\phi_{m i} \phi_{l i} \phi_{m k}^{*} \phi_{l k}^{*}}{a_{i} a_{k}^{*}} \eta_{4}^{(i, k)}\right)\right] .
$$

Based on this, the $k$ th modal dispersion is now defined as follows.

Definition. The $k t h$ modal dispersion is defined as the $k$ th mode's contribution to the vibration signal energy given by the expression

$$
D_{k}(0) \triangleq 2 R_{f_{0}} \sum_{i=1}^{n} \operatorname{Re}\left(\frac{\phi_{m i} \phi_{l i} \phi_{m k} \phi_{l k}}{a_{i} a_{k}} \eta_{3}^{(i, k)}+\frac{\phi_{m i} \phi_{l i} \phi_{m, k}^{*} \phi_{l k}^{*}}{a_{i} a_{k}^{*}} \eta_{4}^{(i, k)}\right) .
$$

In addition, the dispersion percentage (or normalized dispersion) of the $k$ th mode is defined as the dispersion of that mode normalized by the total vibration signal energy; that is,

$$
\delta_{k} \triangleq \frac{D_{k}(0)}{\sum_{j=1}^{n} D_{j}(0)} \times 100 \% \quad(k=1,2, \ldots, n)
$$

Note that expressions (20) and (21) represent the $k$ th modal dispersion and dispersion percentage, respectively, for the $m l$ th transfer function. The additional subscripts indicating this have been dropped for the sake of simplicity, but should be used in cases of potential ambiguity.

The dispersion percentage of a given mode within a particular transfer function therefore constitutes a "measure" of that mode's relative significance within that transfer function. For an $n$-degree-of-freedom structural system characterized by $n$ modes, an $n \times n$ transfer matrix incorporating all scalar transfer functions may be defined, and the dispersion percentages of a given mode may be correspondingly written in the form of a dispersion percentage matrix.

Example. Consider the two-degree-of-freedom proportionally damped system of Figure 1 , with physical parameters

$$
\mathbf{M}=\left[\begin{array}{rr}
1.0 & 0.0 \\
0.0 & 1.0
\end{array}\right], \quad \mathbf{C}=\left[\begin{array}{rr}
2.5 & -0.8 \\
-0.8 & 2.5
\end{array}\right], \quad \mathbf{K}=\left[\begin{array}{rr}
110.0 & -11.0 \\
-11.0 & 110.0
\end{array}\right]
$$

The modal parameters and complete dispersion analysis results, indicating the importance of each vibrational mode in each one of the system's transfer functions, are given in Table 1 in the form of dispersion percentage matrices.

As it is evident from these results, mode 1 is a heavier contributor to the vibration energy, and hence of greater importance than mode 2 for this particular system. Another interesting observation is that the dispersion of mode 2 is negative in both transfer receptance cases (transfer functions $v_{1} / f_{2}$ and $v_{2} / f_{1}$ ); a phenomenon that deserves further attention and will therefore be discussed in detail in what follows. 


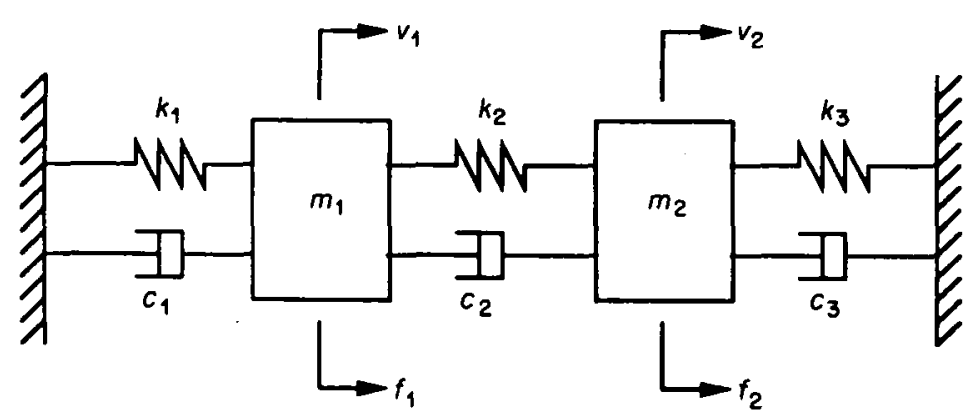

Figure 1. Two-degree-of-freedom structural system.

TABLE 1

Modal parameters and dispersion analysis results for the two-degree-of-freedom proportionally damped system

\begin{tabular}{lcccc}
\hline & $\omega_{n_{k}}(\mathrm{~Hz})$ & $\zeta_{k}$ & $\phi_{k}$ & $\begin{array}{c}\text { Dispersion } \\
\text { percentage matrices } \\
(\%)\end{array}$ \\
\hline Mode 1 & 1.5836 & 0.08543 & $\frac{1}{\sqrt{2}}\left\{\begin{array}{l}1 \\
1\end{array}\right\}$ & {$\left[\begin{array}{rr}61.61 & 132.28 \\
132.28 & 61.61\end{array}\right]$} \\
Mode 2 & 1.7507 & 0.15000 & $\frac{1}{\sqrt{2}}\left\{\begin{array}{r}1 \\
-1\end{array}\right\}$ & {$\left[\begin{array}{rr}38.39 & -32.28 \\
-32.28 & 38.39\end{array}\right]$} \\
\hline
\end{tabular}

\section{MODES CHARACTERIZED BY NEGATIVE DISPERSIONS: THE CASE OF PROPORTIONALLY DAMPED SYSTEMS}

The definition of a structural mode's dispersion, and its interpretation as the mode's contribution to the vibration response energy under broadband stochastic excitation conditions, provides an objective and physically meaningful approach for assessing the relative importance of vibrational modes within a given structural system. However, as it has been already observed, a problem arises in that the dispersions of some modes are negative for certain structural systems, and this creates difficulties, as it is not immediately evident how negative contributions can be interpreted within this context, or what their exact physical significance and role are. As a consequence, and in order for the relative importance of such modes to be properly judged, satisfactory answers to these questions have to be provided.

In achieving this objective alternative interpretations of the notion of modal dispersion and the dispersion analysis methodology, allowing for additional insight into the underlying physical mechanisms, will be now sought. In this section our attention will be focused on the special, but interesting, case of proportionally damped systems, for which somewhat simpler and easier to interpret expressions can be derived. The more general non-proportionally damped case will be examined in section 4 .

Based on expression (20), the contribution $D_{k}(0)$ of the $k$ th mode in the vibration signal energy may be decomposed as

$$
D_{k}(0)=\beta_{k k}+\beta_{k},
$$


where

$$
\begin{gathered}
\beta_{k k} \triangleq 2 R_{f_{0}} \operatorname{Re}\left(\frac{\phi_{m k}^{2} \phi_{l k}^{2}}{a_{k}^{2}} \eta_{3}^{(k, k)}+\frac{\left|\phi_{m k}\right|^{2}\left|\phi_{l k}\right|^{2}}{\left|a_{k}\right|^{2}} \eta_{4}^{(k, k)}\right), \\
\beta_{k} \triangleq \sum_{i=1, i \neq k}^{n} \beta_{i k}, \\
\beta_{i k} \triangleq 2 R_{f_{0}} \operatorname{Re}\left(\frac{\phi_{m i} \phi_{l i} \phi_{m k} \phi_{l k}}{a_{i} a_{k}} \eta_{3}^{(i, k)}+\frac{\phi_{m i} \phi_{l i} \phi_{l m k}^{*} \phi_{l k}^{*}}{a_{i} a_{k}^{*}} \eta_{4}^{(i, k)}\right) .
\end{gathered}
$$

The term $\beta_{k k}$ in this decomposition depends upon the $k$ th modal parameters and will be therefore referred to as the $k$ th modal autocorrelation, whereas the term $\beta_{k}$ depends upon the remaining modal parameters as well, and will be referred to as the $k t h$ modal crosscorrelation. This latter term represents the part of the energy due to the interactions between the $k$ th mode and the rest of the modes present in the system.

In the special case of proportionally damped structural systems the above expressions reduce to $\dagger$

$$
\begin{gathered}
\beta_{k k}=\frac{R_{f_{0}} \phi_{m k}^{2} \phi_{l k}^{2}}{4 \zeta_{k} \omega_{n_{k}}^{3}}, \\
\beta_{k}=\sum_{i=1, i \neq k}^{n} \frac{2 R_{f_{0}} \phi_{m i} \phi_{l i} \phi_{m k} \phi_{l k}\left(\zeta_{i} \omega_{n_{i}}+\zeta_{k} \omega_{n_{k}}\right)}{\left(\omega_{n_{i}}^{2}-\omega_{n_{k}}^{2}\right)^{2}+4\left[\zeta_{i} \zeta_{k} \omega_{n_{i}} \omega_{n_{k}}\left(\omega_{n_{i}}^{2}+\omega_{n_{k}}^{2}\right)+\left(\zeta_{i}^{2}+\zeta_{k}^{2}\right) \omega_{n_{i}}^{2} \omega_{n_{k}}^{2}\right]},
\end{gathered}
$$

where $\phi_{i}$ represents the $i$ th mass-normalized eigenvector, and the fact that the $a_{i}$ 's of equations (7) and (8) can, in this case, be simply expressed as $a_{i}=\mathrm{j} 2 \omega_{d_{i}}(i=1,2, \ldots, n)$, was used. Based on these expressions the following corollaries follow.

Corollary 1 . The modal autocorrelation is non-negative for any mode of a proportionally damped system. The dispersion of any particular mode will thus be negative if and only if its modal cross-correlation is negative and of magnitude larger than that of its modal autocorrelation.

Corollary 2. In a proportionally damped system no negative dispersion modes can occur within a point receptance transfer function (that is, a transfer function relating identical excitation $l$ and response $m$ co-ordinates). However, negative dispersion modes can be encountered in transfer receptance cases, in which the excitation and response co-ordinates are different $(l \neq m)$.

Corollary 3. A necessary condition for the $k$ th mode to be characterized by negative dispersion within the $m l$ th $(m \neq l)$ transfer function of a proportionally damped system is that at least one of the products of mode shape elements $\phi_{m i} \phi_{l i} \phi_{m k} \phi_{l k}$ for $i=1,2, \ldots, n$ be negative.

As it will be shown later, the validity of Corollary 1 can be extended to the case of nonproportionally damped systems as well.

In order to gain further insight into this problem, the natural frequency ratio between two modes is defined as

$$
\varepsilon_{\omega_{i k}} \triangleq \omega_{n_{i}} / \omega_{n_{k}}
$$

$\dagger$ These expressions are analogous to those in Elishakoff [26], who distinguishes modal autocorrelation and overall cross-correlation terms in the total vibration energy of proportionally damped systems. 
Then, the $k$ th modal cross-correlation $\beta_{k}$ of the proportionally damped structural system [equation (27)] may be written as

$$
\beta_{k}=\sum_{i=1, i \neq k}^{n} \frac{2 R_{f_{0}} \phi_{m i} \phi_{l i} \phi_{m k} \phi_{l k}\left(\zeta_{i} \varepsilon_{\omega_{i k}}+\zeta_{k}\right)}{\omega_{n_{k}}^{3}\left[\left(\varepsilon_{\omega_{i k}}^{2}-1\right)^{2}+4\left[\zeta_{i} \zeta_{k} \varepsilon_{\omega_{i k}}\left(\varepsilon_{\omega_{\text {lik }}}^{2}+1\right)+\left(\zeta_{i}^{2}+\zeta_{k}^{2}\right) \varepsilon_{\omega_{l k}}^{2}\right]\right]} .
$$

It is interesting to observe that both the modal autocorrelation $\beta_{k k}$ [equation (26)] and cross-correlation $\beta_{k}$ [equation (29)] have the term $\omega_{n_{k}}^{3}$ as a common factor in their denominators. By using appropriate natural frequency ratios, the quantity $\omega_{n_{k}}^{3}$ may thus be made a multiplicative factor in the total vibration signal energy expression and, as a consequence, the dispersion percentages $\delta_{k}(k=1,2, \ldots, n)$ calculated from equation (21) will be independent of it; in fact, independent of any one of the exact natural frequency values. This observation is organized into the following corollary.

Corollary 4 . The $k$ th mode dispersion percentage $\delta_{k}$ of a proportionally damped structural system does not depend upon the exact value of any one of the system's natural frequencies $\omega_{n_{i}}(i=1, \ldots, n)$, but only upon the natural frequency ratios $\varepsilon_{\omega_{\text {uk }}}(i=1, \ldots, n)$.

For the rest of this section we will further limit our analysis to the case of proportionally damped systems satisfying the following assumptions: (A1) the structural system is lightly damped $\left(\zeta_{i} \ll 1, \forall i\right)$; (A2) the mass-normalized mode shape elements are all of the same order of magnitude.

Apart from being of obvious practical importance, this case allows for some additional calculations that provide insight into the physical conditions under which negative dispersion modes may appear. Towards this end let us examine the dispersion percentage $\delta_{k}$ as a function of the natural frequency ratios $\varepsilon_{\omega_{i k}}(i=1,2, \ldots, n)$.

If $\varepsilon_{a_{k j}} \gg 1, \forall i, i \neq k$, the terms (i) and (ii) in expression (29) may be approximately expressed as

$$
\text { (i) } \approx \varepsilon_{\omega_{i k}}^{4}, \quad \text { (ii) } \approx 4 \zeta_{i} \zeta_{k} \varepsilon_{\omega_{i k}}^{3},
$$

and thus (i) $\gg$ (ii); an inequality further strengthened by the light damping assumption $\mathrm{Al}$.

If, on the other hand, $\varepsilon_{w_{k}} \ll 1$, then by neglecting terms of order higher than two we have

$$
\text { (i) } \approx 1, \quad \text { (ii) } \approx 4 \zeta_{i} \zeta_{k} \varepsilon_{\omega_{i k}},
$$

and (i) $\gg$ (ii) in this case as well.

As a consequence, if the lightly damped structural system has well-separated modes ( $\varepsilon_{\omega_{i k}} \gg 1$ or $\varepsilon_{\omega_{i k}} \ll 1, \forall i, i \neq k$ ), the term (i) is dominant to (ii), and the $k$ th mode dispersion $D_{k}(0)$ may be thus approximated as [see equations (26) and (27)]

$$
D_{k}(0)=\beta_{k k}+\beta_{k} \approx \frac{R_{f_{0}}}{\omega_{n_{k}}^{3}}\left[\frac{\phi_{m k}^{2} \phi_{l k}^{2}}{4 \zeta_{k}}+\sum_{i=1, i \neq k}^{n} \frac{2 \phi_{m i} \phi_{l i} \phi_{m k} \phi_{l k}\left(\zeta_{i} \varepsilon_{\omega_{i k}}+\zeta_{k}\right)}{\left(\varepsilon_{\omega_{i k}}^{2}-1\right)^{2}}\right],
$$

with the denominator of (iv) further approximated as $\varepsilon_{\omega_{i k}}^{4}$ (if $\varepsilon_{\omega_{i k}} \gg 1$ ) or 1 (if $\varepsilon_{\boldsymbol{o}_{\text {lk }}} \ll 1$ ). In both cases, however, and under assumptions $A 1$ and $A 2$, the term (iii) will be dominant, in magnitude, to (iv). Due to the difference in magnitude between these two terms, and for reasonably small number of degrees of freedom $n$, (iii) will be dominant when compared to the sum of the terms (iv) as well. That is, the modal autocorrelation will be significantly larger, in magnitude, than the modal cross-correlation, and we, therefore, arrive at the following corollary. 
Corollary 5. For a proportionally damped system satisfying $A 1$ and $A 2$, and characterized by well-separated modes $\left(\varepsilon_{\omega_{\mu k}} \gg 1\right.$ or $\left.\varepsilon_{\omega_{i k}} \ll 1, \forall i, i \neq k\right)$, the modal autocorrelation terms dominate, in magnitude, the corresponding modal cross-correlations, and thus, by virtue of Corollary 1, no negative dispersion modes exist.

Based on this result, it is evident that a necessary condition for the $k$ th mode of a proportionally damped structural system satisfying A1 and A2 to be characterized by negative dispersion is that at least one additional natural frequency be close to $\omega_{n_{k}}$, so that the term (ii) in equation (29) can dominate (i), and the modal autocorrelation $\beta_{k k}$ does not necessarily dominate (in magnitude) in equation (32). This is a somewhat expected result, since for $\delta_{k}$ to be negative the $k$ th modal cross-correlation needs to be negative and of magnitude larger than that of its modal autocorrelation (Corollary 1), with the former (modal cross-correlation) becoming maximum as another damped natural frequency approaches $\omega_{d_{k}}\left(\omega_{d_{1}} \rightarrow \omega_{d_{k}}\right)$ (see last remark in subsection 4.1$)$; a fact that, in the case of lightly damped systems, occurs only when $\omega_{n,}$ approaches $\omega_{n_{k}}$. This behavior of the modal cross-correlation is in agreement with a related observation in reference [26], that in a two-degree-of-freedom system the contribution of the overall cross-correlation term to the total vibration energy becomes significant as the two natural frequencies crowd together.

In order to investigate how close two modes should be in order for the term (i) not to necessarily dominate in the denominator of expression (29), the special case of a twodegree-of-freedom system is now considered. By defining the quantity $\Delta$ through the expression

$$
\varepsilon_{\omega_{12}} \triangleq 1+\Delta, \quad \Delta \ll 1,
$$

and substituting it into the terms (i) and (ii) of equation (29), we obtain

$$
\begin{gathered}
\left(\text { i) }=\left((1+\Delta)^{2}-1\right)^{2}=4 \Delta^{2}+4 \Delta^{3}+\Delta^{4},\right. \\
\text { (ii) }=4\left[\zeta_{1} \zeta_{2}(1+\Delta)\left[(1+\Delta)^{2}+1\right]+\left(\zeta_{1}^{2}+\zeta_{2}^{2}\right)(1+\Delta)^{2}\right] \\
=4\left[\left(\zeta_{1}+\zeta_{2}\right)^{2}+\zeta_{1} \zeta_{2}\left(4 \Delta+4 \Delta^{2}+\Delta^{3}\right)+\left(\zeta_{1}^{2}+\zeta_{2}^{2}\right)\left(2 \Delta+\Delta^{2}\right)\right] .
\end{gathered}
$$

By neglecting terms involving orders of $\Delta$ higher than two, and noting that, in view of A1, the terms $16 \zeta_{1} \zeta_{2} \Delta^{2}$ and $4\left(\zeta_{1}^{2}+\zeta_{2}^{2}\right) \Delta^{2}$, are also negligible, we have

$$
\text { (i) } \approx 4 \Delta^{2}, \quad \text { (ii) } \approx 4\left(\zeta_{1}+\zeta_{2}\right)^{2}+16 \zeta_{1} \zeta_{2} \Delta+4\left(\zeta_{1}^{2}+\zeta_{2}^{2}\right) \Delta^{2} .
$$

In order for term (ii) to be dominant,

$$
4 \Delta^{2}<4\left(\zeta_{1}+\zeta_{2}\right)^{2}+16 \zeta_{1} \zeta_{2} \Delta+4\left(\zeta_{1}^{2}+\zeta_{2}^{2}\right) \Delta^{2} \Leftrightarrow \Delta^{2}-2\left(\zeta_{1}+\zeta_{2}\right)^{2} \Delta-\left(\zeta_{1}+\zeta_{2}\right)^{2}<0,
$$

or, equivalently,

$$
\Delta_{2} \equiv\left(\zeta_{1}+\zeta_{2}\right)^{2}-\sqrt{\left(\zeta_{1}+\zeta_{2}\right)^{4}+\left(\zeta_{1}+\zeta_{2}\right)^{2}}<\Delta<\left(\zeta_{1}+\zeta_{2}\right)^{2}+\sqrt{\left(\zeta_{1}+\zeta_{2}\right)^{4}+\left(\zeta_{1}+\zeta_{2}\right)^{2}} \equiv \Delta_{1},
$$

where, obviously, $\Delta_{1}>0$ and $\Delta_{2}<0$. By approximating the quantity under the square root as $\left(\zeta_{1}+\zeta_{2}\right)^{2}$, and neglecting terms of order higher than two, equation (39) may be simplified as

$$
|\Delta|<\zeta_{1}+\zeta_{2}
$$

Expression (39), or its approximate version (40), therefore composes a necessary condition for a negative dispersion mode to appear in a two-degree-of-freedom proportionally damped system satisfying A1 and A2. As is evident from this condition, the lighter the 
damping of the system, the smaller the magnitude of $\Delta$ (and thus the smaller the distance between the two natural frequencies) that is necessary for a negative dispersion mode to appear. These facts are illustrated in the example that follows.

Example (continued). Consider the two-degree-of-freedom structural system of Figure 1. With mode shapes fixed (same as in Table 1), the dispersion percentages of the transfer function $v_{2} / f_{1}$ are shown in Figure 2 as functions of the natural frequency ratio $\varepsilon_{\omega_{12}}$ for four cases, as follows: (a) $\zeta_{1}=0.35, \zeta_{2}=0.5$; (b) $\zeta_{1}=0.07, \zeta_{2}=0.1 ;$ (c) $\zeta_{1}=0.007 ; \zeta_{2}=$ 0.01 ; (d) $\zeta_{1}=0.0007, \zeta_{2}=0.001$. As is evident from this figure, the lighter the damping of the system, the closer the two natural frequencies should be in order for a negative dispersion mode to occur. Although condition (40) is, of course, not applicable to the high damping case (a), it is easy to verify that it is satisfied in all of the remaining cases for the intervals of $\Delta$ for which a negative dispersion mode occurs. Indeed, in case (b) mode 2 has negative dispersion for $0.9<\varepsilon_{\omega_{12}}<1.05(-0.1<\Delta<0.5)$, whereas $\zeta_{1}+\zeta_{2}=0 \cdot 17$. Similarly, in case (c), mode 2 has negative dispersion for $0.993<\varepsilon_{\omega_{12}}<1.006$

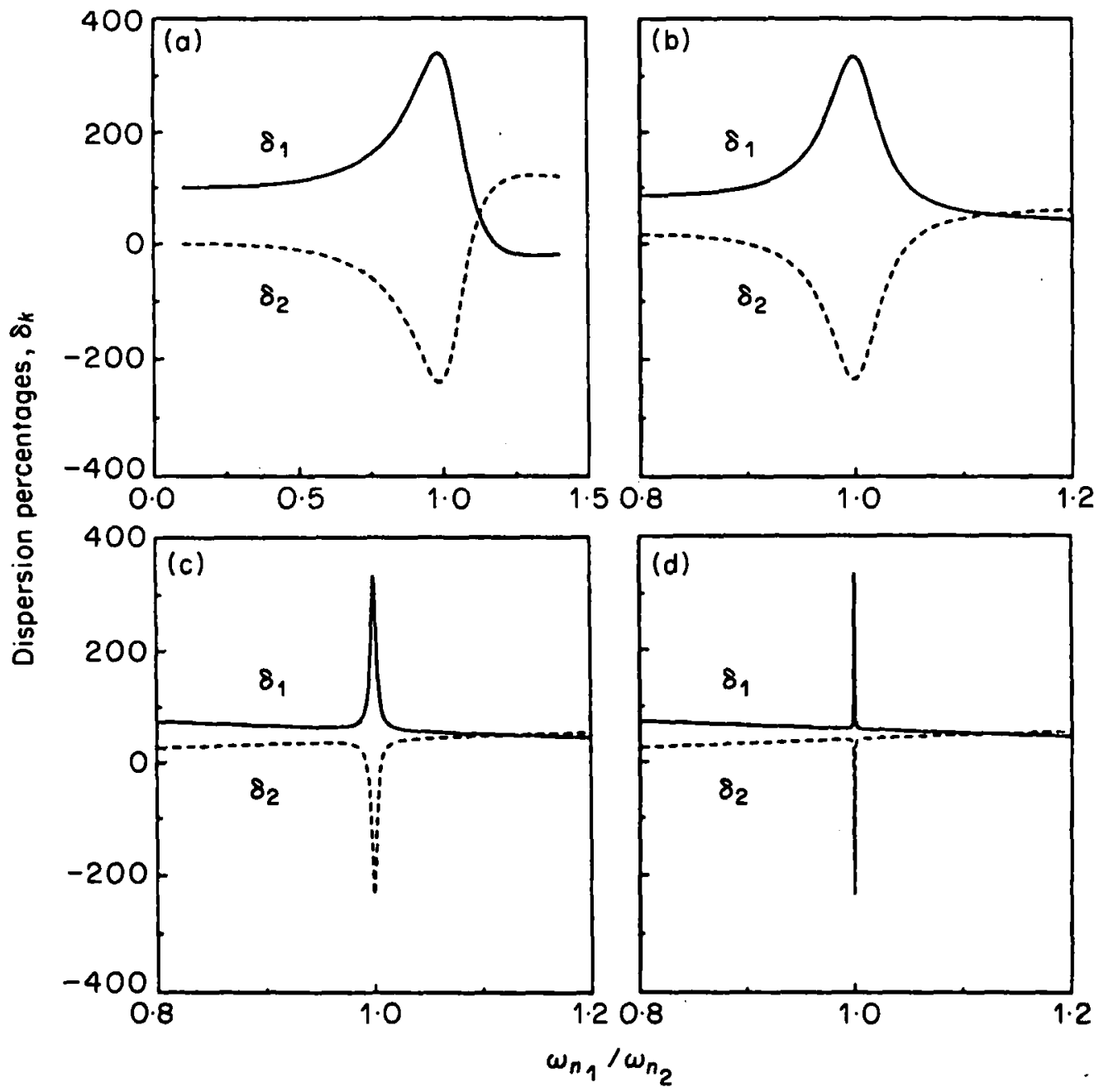

Figure 2. The dispersion percentage of a proportionally damped system as a function of the natural frequency ratio, $\varepsilon_{\omega_{12}}=\omega_{n_{1}} / \omega_{n_{2}}:-$, mode $1 ;-\cdots$, mode 2 . (a) $\zeta_{1}=0.35, \zeta_{2}=0.50 ;$ (b) $\zeta_{1}=0.07, \zeta_{2}=0 \cdot 10 ;$ (c) $\zeta_{1}=0.007$, $\zeta_{2}=0.01$; (d) $\zeta_{1}=0.0007, \zeta_{2}=0.001$. Two-degree-of-freedom system; transfer function $v_{2} / f_{1}$. 
$(-0.007<\Delta<0.006)$, and $\zeta_{1}+\zeta_{2}=0.017$, and in case (d), mode 2 has negative dispersion for $0.9994<\varepsilon_{\omega_{12}}<1.0006(-0.0006<\Delta<0.0006)$ and $\zeta_{1}+\zeta_{2}=0.0017$.

\section{INTERPRETATIONS AND PHYSICAL SIGNIFICANCE OF DISPERSION ANALYSIS: THE GENERAL NON-PROPORTIONALLY DAMPED SYSTEM CASE}

In this section the non-proportionally damped system case is considered, and various interpretations of the notion of modal dispersion are derived. These offer additional physical insight into the proposed dispersion analysis methodology and further clarify the issue of negative dispersion modes.

\subsection{INTERPRETATION IN TERMS OF MODAL IMPULSE RESPONSE FUNCTIONS}

A key interpretation in this context is based on the currently introduced notion of modal impulse response function for general viscously damped structural systems. For this purpose consider the function $x(t)$, defined as

$$
x(t) \triangleq \sum_{i=1}^{n}\left[\frac{\phi_{m i} \phi_{l i}}{a_{i}} h_{i}(t)+\frac{\phi_{m i}^{*} \phi_{l i}^{*}}{a_{i}^{*}} h_{i}^{*}(t)\right]
$$

From the examination of the response equation (4) of a non-proportionally damped structural system, it can be shown that $x(t)$ may be interpreted as the impulse response function of the $m l$ th transfer function and be decomposed as

$$
x(t) \triangleq \sum_{i=1}^{n}\left[z_{i}(t)+z_{i}^{*}(t)\right]=\sum_{i=1}^{n} 2 \operatorname{Re}\left[z_{i}(t)\right] \triangleq \sum_{i=1}^{n} x_{i}(t)
$$

with obvious definitions for $z_{i}(t)$ and $x_{i}(t)(i=1,2, \ldots, n)$. From these expressions it is evident that $x_{i}(t)$ is real-valued and represents that part of the impulse response function $x(t)$ that is associated with the $i$ th vibrational mode, and will be thus referred to as the ith modal impulse response function within the $m l$ th transfer function.

Based on this, the vibration signal energy given by equation (16) may be rewritten as

$$
\begin{aligned}
R_{v_{m}}(0) & =\sum_{k=1}^{n}\left[2 R_{f_{0}} \sum_{i=1}^{n} \int_{0}^{\infty} \operatorname{Re}\left[z_{i}(t) z_{k}(t)+z_{i}(t) z_{k}^{*}(t)\right] \mathrm{d} t\right] \\
& =\sum_{k=1}^{n}\left[4 R_{f_{0}} \sum_{i=1}^{n} \int_{0}^{\infty} \operatorname{Re}\left[z_{i}(t)\right] \operatorname{Re}\left[z_{k}(t)\right] \mathrm{d} t\right] \\
& =\sum_{k=1}^{n}\left[R_{f_{0}} \sum_{i=1}^{n} \int_{0}^{\infty} x_{i}(t) x_{k}(t) \mathrm{d} t\right],
\end{aligned}
$$

and the contribution $D_{k}(0)$ of the $k$ th vibrational mode to the total vibration signal energy may be expressed as

$$
D_{k}(0) \triangleq \beta_{k k}+\beta_{k}=R_{f_{0}} \int_{0}^{\infty} x_{k}^{2}(t) \mathrm{d} t+R_{f_{0}} \sum_{i=1, i \neq k}^{n} \int_{0}^{\infty} x_{i}(t) x_{k}(t) \mathrm{d} t .
$$

From this form it is evident that the $k$ th modal autocorrelation $\beta_{k k}$ is proportional to the deterministic autocorrelation function of the $k$ th modal impulse response $\left\{x_{k}(t)\right\}$ evaluated at lag $\tau=0$, and that the $k$ th modal cross-correlation $\beta_{k}$ is proportional to the sum of the 
deterministic cross-correlation functions between $\left\{x_{k}(t)\right\}$ and $\left\{x_{i}(t)\right\}(i=1,2, \ldots, n ; i \neq k)$, also evaluated at lag $\tau=0$. The coefficient of proportionality is, in both cases, equal to the intensity of the uncorrelated stochastic excitation signal. Based on these observations, the following corollary (that is an extension of Corollary 1 to the case of non-proportionally damped structures) follows.

Corollary 6. All modal autocorrelations are necessarily non-negative. As a consequence, in order for the $k$ th vibrational mode to have negative dispersion, its modal cross-correlation has to be negative and of magnitude larger than that of its modal autocorrelation.

Based on equation (44), the contribution of the $k$ th mode to the vibration signal energy may alternatively be expressed as

$$
D_{k}(0)=R_{f_{0}} \sum_{i=1}^{n} \int_{0}^{\infty} x_{i}(t) x_{k}(t) \mathrm{d} t=R_{f_{0}} \int_{0}^{\infty} x(t) x_{k}(t) \mathrm{d} t,
$$

and the corresponding dispersion percentage rewritten in the form

$$
\delta_{k}=\frac{\int_{0}^{\infty} x(t) x_{k}(t) \mathrm{d} t}{\int_{0}^{\infty} x^{2}(t) \mathrm{d} t} \times 100 \%
$$

The following interpretations in terms of deterministic correlation functions are now immediate.

Corollary 7. The contribution (dispersion) of the $k$ th mode to the vibration signal energy is equal to the product of the uncorrelated stochastic excitation intensity and the deterministic cross-correlation between the total impulse response $\{x(t)\}$ and the modal impulse response $\left\{x_{k}(t)\right\}$ evaluated at lag $\tau=0$. The corresponding dispersion percentage is hence equal to the cross-correlation between $\{x(t)\}$ and $\left\{x_{k}(t)\right\}$ at lag zero, normalized by the autocorrelation of $\{x(t)\}$ at that same lag.

Corollary 8. A mode characterized by negative dispersion has an associated modal impule response function $\left\{x_{k}(t)\right\}$ that is negatively correlated with the total impulse response $\{x(t)\}$ in the time interval $[0, \infty)$. This means that the $k$ th mode is, on average, acting in a way that opposes $\{x(t)\}$, and therefore reduces its magnitude.

Remark. The degree of cross-correlatedness between $\{x(t)\}$ and $\left\{x_{k}(t)\right\}$, that determines the $k$ th modal dispersion, may also be assessed in terms of the quantity

$$
\rho_{k}=\frac{\int_{0}^{\infty} x(t) x_{k}(t) \mathrm{d} t}{\sqrt{\int_{0}^{\infty} x^{2}(t) \mathrm{d} t \int_{0}^{\infty} x_{k}^{2}(t) \mathrm{d} t}},
$$

which, due to the Cauchy-Buniakovski inequality [27], is normalized in the interval $[-1,1]$.

These ideas are now illustrated through an example.

Example (continued). Consider the two-degree-of-freedom proportionally damped system of Figure 1 with modal parameters and dispersion analysis results presented in Table 1. As it may be observed, and in accordance with Corollary 2, the dispersion percentages are both positive in the point receptance cases (transfer functions $v_{1} / f_{1}$ and $v_{2} / f_{2}$ ), but of opposite signs in the transfer receptance cases (transfer functions $v_{2} / f_{1}$ and $v_{1} / f_{2}$ ). The transfer receptance case $v_{2} / f_{1}$ is further examined in Figure 3. The modal impulse responses $x_{1}(t)$ and $x_{2}(t)$ are shown in part (a) of the figure, and are apparently negatively correlated 


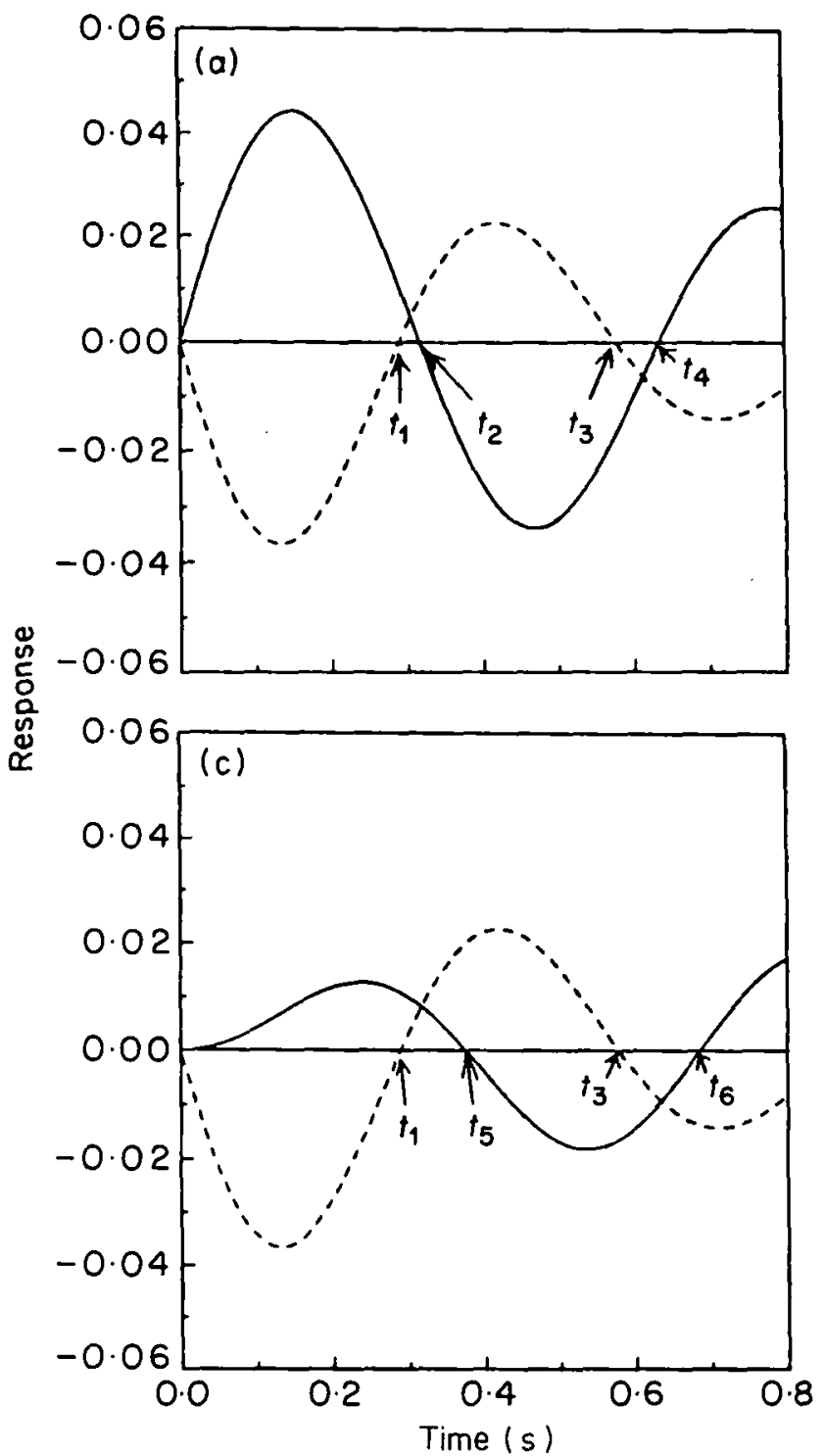

Figure 3. (a) The first $\left[x_{1}(t)\right],(-)$ and second $\left[x_{2}(t)\right],(--)$ modal impulse response functions; (b) the products $x_{1}(t) x_{2}(t),(-)$ and $x_{2}^{2}(t),(---) ;\left(\right.$ c) the total $[x(t)],(-)$ and second modal $\left[x_{2}(t)\right],(---)$ impulse response functions; (d) the product $x(t) x_{2}(t)$. Two-degree-of-freedom system; transfer function $v_{2} / f_{1}$.

in the observation time interval. Indeed, the second modal cross-correlation is (assuming that $R_{f_{0}}=1$ )

$$
\begin{aligned}
\beta_{12} & =\int_{0}^{\infty} x_{1}(t) x_{2}(t) \mathrm{d} t \\
& =\underbrace{\int_{0}^{t_{1}} x_{1}(t) x_{2}(t) \mathrm{d} t}_{\gamma_{1}}+\underbrace{\int_{t_{1}}^{t_{2}} x_{1}(t) x_{2}(t) \mathrm{d} t}_{\gamma_{2}}+\underbrace{\int_{t_{2}}^{t_{3}} x_{1}(t) x_{2}(t) \mathrm{d} t+\cdots}_{\gamma_{3}}
\end{aligned}
$$




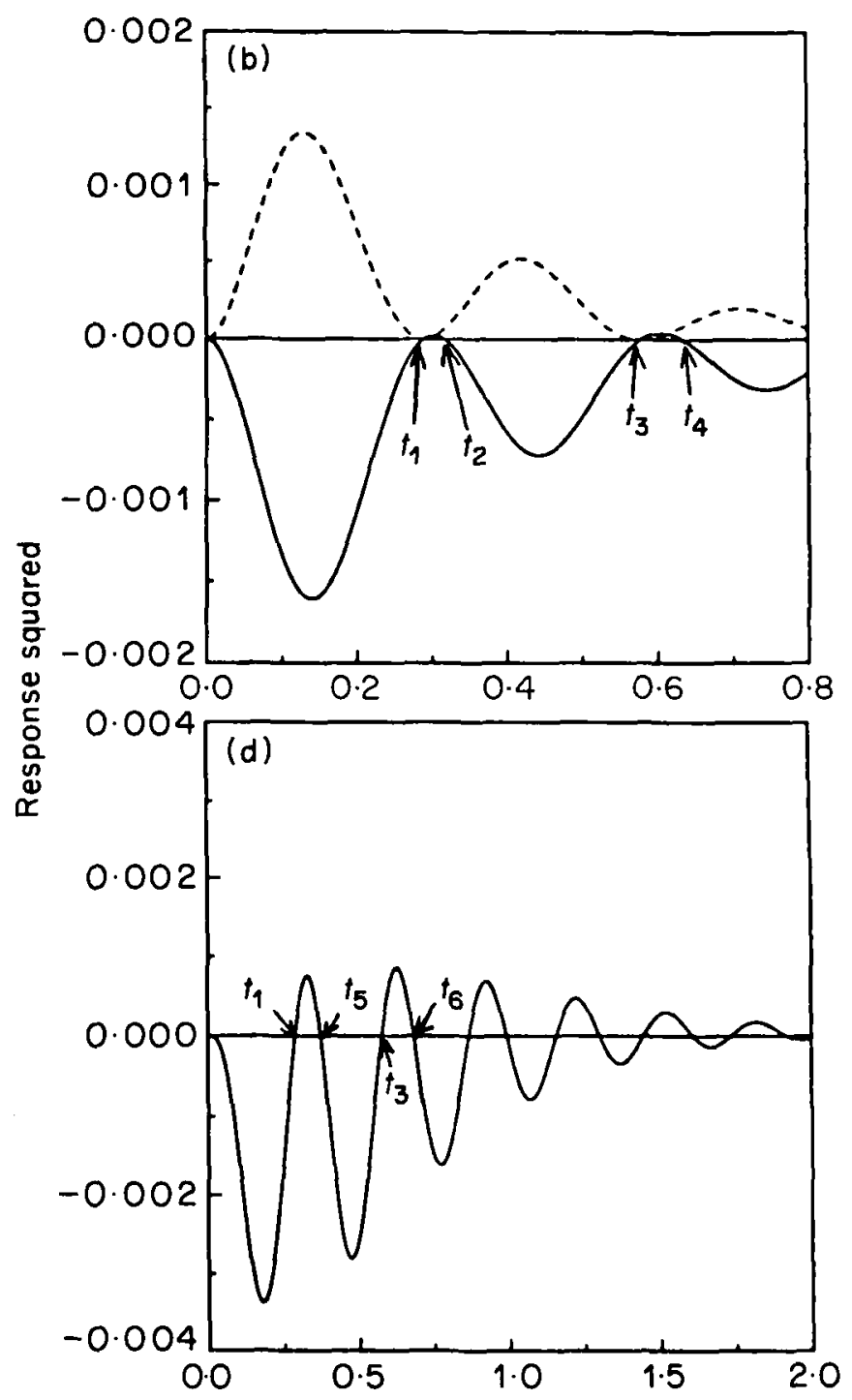

Figure 3-continued.

In the first time interval $\left[0, t_{1}\right]$ the modal cross-correlation is negative $\left(\gamma_{1}<0\right)$, then (time interval $\left.\left[t_{1}, t_{2}\right]\right)$ positive $\left(\gamma_{2}>0\right)$, and so on. As time grows, the impulse responses decay, and their overall (in the time interval $[0, \infty)$ ) cross-correlation is negative. This is also illustrated in part (b) of the figure, where the curves $x_{1}(t) x_{2}(t)$ and $x_{2}^{2}(t)$ are shown. The area between the former curve and the time axis represents the modal cross-correlation term $\left(\beta_{2}=\beta_{12}\right)$, whereas the area between the latter curve and the time axis represents the second modal autocorrelation term $\left(\beta_{22}\right)$. It is apparent that $\beta_{2}+\beta_{22}<0$, so that the second mode is characterized by negative dispersion. Similar observations may be made from parts (c) and (d) of the figure, which depict the impulse responses $x(t), x_{2}(t)$ and the product $x(t) x_{2}(t)$, respectively. Indeed, it is evident that the two responses are negatively correlated, so that on average (in the time interval $[0, \infty)$ ) $x_{2}(t)$ tends to suppress $x(t)$. 
Remark. Based on the discussion thus far and the above example, it is evident that for general (non-proportionally damped) structural systems with adjacent damped natural frequencies $\left(\omega_{d_{1}} \approx \omega_{d_{2}}\right.$ ), one mode will necessarily have negative contribution (dispersion), provided that the modal impulse responses $\left\{x_{1}(t)\right\}$ and $\left\{x_{2}(t)\right\}$ start out in opposite directions. In addition, it is also evident that the modal cross-correlation has maximum effects when $\omega_{d_{1}}=\omega_{d_{2}}$.

\subsection{INTER PRETATION IN TERMS OF MODAL RESPONSES DUE TO UNCORRELATED STOCHASTIC EXCITATION}

Due to the duality between the deterministic delta function and uncorrelated stochastic signals, it is expected that the foregoing results and interpretations should be (appropriately) extendable to the case of stochastic force excitation by considering corresponding modal responses. That is indeed the case, and may be shown as follows.

Consider a structural system excited by an uncorrelated stochastic excitation $\left\{f_{1}(t)\right\}$ and define the part of the response associated with mode $k$, referred to as the kth modal response, by the convolution integral

$$
v_{m_{k}}(t) \triangleq \int_{-\infty}^{t} x_{k}(t-\tau) f_{l}(\tau) \mathrm{d} \tau .
$$

The total system response may be similarly expressed as

$$
v_{m}(t)=\int_{-\infty}^{t} x(t-\tau) f_{l}(\tau) \mathrm{d} \tau,
$$

and the cross-correlation between the signals $\left\{v_{m}(t)\right\}$ and $\left\{v_{m_{k}}(t)\right\}$ at lag $\tau=0$ is then

$$
\begin{aligned}
\mathrm{E}\left[v_{m}(t) v_{m_{k}}(t)\right] & =\int_{-\infty}^{t} \int_{-\infty}^{t} x\left(t-\tau_{1}\right) x_{k}\left(t-\tau_{2}\right) \mathrm{E}\left[f_{l}\left(\tau_{1}\right) f_{l}\left(\tau_{2}\right)\right] \mathrm{d} \tau_{1} \mathrm{~d} \tau_{2} \\
& =\int_{-\infty}^{t} x\left(t-\tau_{1}\right) x_{k}\left(t-\tau_{1}\right) R_{f_{0}} \mathrm{~d} \tau_{1} \\
& =R_{f_{0}} \int_{0}^{\infty} x(\lambda) x_{k}(\lambda) \mathrm{d} \lambda .
\end{aligned}
$$

By comparing this expression to equation (45), the following interpretation in terms of stochastic cross-correlation functions is obtained.

Corollary 9. The contribution (dispersion) of the $k$ th mode to the vibration signal energy is equal to the cross-correlation between the corresponding total vibration response and the $k$ th modal response evaluated at lag zero; that is

$$
D_{k}(0)=\mathrm{E}\left[v_{m}(t) v_{m_{k}}(t)\right]
$$

In the case that mode $k$ is characterized by negative dispersion, $\mathrm{E}\left[v_{m}(t) v_{m_{k}}(t)\right]$ will be negative, so that at every time $t$ the $k$ th mode is, in an ensemble average sense, acting in a way that opposes the total response $\left\{v_{m}(t)\right\}$, and therefore reduces its magnitude.

\subsection{SPECTRAL INTER PRETATION}

The notion of modal dispersion is now examined in the spectral domain. Towards this end define as $F_{k}(j \omega)$ the Fourier transform of the part of the output autocovariance 
associated with the $k$ th vibrational mode

$$
F_{k}(\mathrm{j} \omega) \triangleq \frac{1}{2 \pi} \int_{-\infty}^{\infty} D_{k}(\tau) \mathrm{e}^{-\mathrm{j} \omega \tau} \mathrm{d} \tau \quad(k=1,2, \ldots, n) .
$$

The spectrum of the vibration signal $\left\{v_{m}(t)\right\}$ may be then expressed in the decomposition form

$$
S_{v_{m}}(\omega)=\frac{1}{2 \pi} \int_{-\infty}^{\infty} R_{v_{m}}(\tau) \mathrm{e}^{-\mathrm{j} \omega \tau} \mathrm{d} \tau=\sum_{k=1}^{n} F_{k}(\mathrm{j} \omega) \Rightarrow S_{v_{m}}(\omega)=\sum_{k=1}^{n} S_{k}(\omega)
$$

where $S_{k}(\omega)$ is defined as the real part of $F_{k}(\mathrm{j} \omega)$ :

$$
S_{k}(\omega) \triangleq \operatorname{Re}\left[F_{k}(\mathrm{j} \omega)\right]
$$

Based on these expressions it is evident that $S_{k}(\omega)$ represents the contribution of the $k$ th vibrational mode to the vibration signal energy at each particular frequency $\omega$, and will be thus referred to as the spectral contribution of the $k$ th mode. The relationship between the modal dispersion and $S_{k}(\omega)$ may be readily established by considering the inverse Fourier transform of equation (52) for $\tau=0$

$$
D_{k}(0)=\int_{-\infty}^{\infty} S_{k}(\omega) \mathrm{d} \omega
$$

The dispersion of the $k$ th vibrational mode is thus equal to the integral of its spectral contribution; that is, the area between the latter curve and the frequency axis. $D_{k}(0)$ will therefore be negative exactly when the integral of $S_{k}(\omega)$ is negative, and the following interpretation of negative dispersion modes follows.

Corollary 10. A mode is characterized by negative dispersion if and only if its overall [in the frequency range $(-\infty, \infty)$ ] contribution to the power spectrum of the vibration signal produced by an uncorrelated stochastic excitation is negative. This result is complementary to the time-domain results of Corollaries 8 and 9 , and indicates that a negative dispersion mode acts in a way that suppresses the spectrum of the vibration signal, and therefore its energy, in the frequency range $(-\infty, \infty)$.

For the computation of the spectral contribution $S_{k}(\omega)$ of the $k$ th mode to the vibration signal energy in the general case of a non-proportionally damped structural system, we proceed as follows. Rewrite the modal contribution $D_{k}(\tau)$ to the vibration signal autocovariance in the form [see equations (9), (10) and (13)]

$$
\begin{aligned}
D_{k}(\tau)= & \sum_{i=1}^{n}\left(\frac{\phi_{m i} \phi_{l i} \phi_{m k} \phi_{l k}}{a_{i} a_{k}} \int_{-\infty}^{\infty} \int_{-\infty}^{\infty} R_{f}\left(\tau+\tau_{1}-\tau_{2}\right) h_{i}\left(\tau_{1}\right) h_{k}\left(\tau_{2}\right) \mathrm{d} \tau_{1} \mathrm{~d} \tau_{2}\right. \\
& +\frac{\phi_{m i} \phi_{l i} \phi_{m k}^{*} \phi_{l k}^{*}}{a_{i} a_{k}^{*}} \int_{-\infty}^{\infty} \int_{-\infty}^{\infty} R_{f}\left(\tau+\tau_{1}-\tau_{2}\right) h_{i}\left(\tau_{1}\right) h_{k}^{*}\left(\tau_{2}\right) \mathrm{d} \tau_{1} \mathrm{~d} \tau_{2} \\
& +\frac{\phi_{m i}^{*} \phi_{l i}^{*} \phi_{m k} \phi_{l k}}{a_{i}^{*} a_{k}} \int_{-\infty}^{\infty} \int_{-\infty}^{\infty} R_{f}\left(\tau+\tau_{1}-\tau_{2}\right) h_{i}^{*}\left(\tau_{1}\right) h_{k}\left(\tau_{2}\right) \mathrm{d} \tau_{1} \mathrm{~d} \tau_{2} \\
& \left.+\frac{\phi_{m i}^{*} \phi_{l i}^{*} \phi_{m k}^{*} \phi_{l k}^{*}}{a_{i}^{*} a_{k}^{*}} \int_{-\infty}^{\infty} \int_{-\infty}^{\infty} R_{f}\left(\tau+\tau_{1}-\tau_{2}\right) h_{i}^{*}\left(\tau_{1}\right) h_{k}^{*}\left(\tau_{2}\right) \mathrm{d} \tau_{1} \mathrm{~d} \tau_{2}\right)
\end{aligned}
$$


The Fourier transform of the first term in this expression may be written as

$$
\begin{aligned}
& \frac{\phi_{m i} \phi_{l i} \phi_{m k} \phi_{l k}}{a_{i} a_{k}} \int_{-\infty}^{\infty} \int_{-\infty}^{\infty} h_{i}\left(\tau_{1}\right) h_{k}\left(\tau_{2}\right) d \tau_{1} d \tau_{2} \times \frac{1}{2 \pi} \int_{-\infty}^{\infty} R_{f}\left(\tau+\tau_{1}-\tau_{2}\right) \mathrm{e}^{-\mathrm{j} \omega r} \mathrm{~d} \tau \\
& =\frac{\phi_{m i} \phi_{l i} \phi_{m k} \phi_{l k}}{a_{i} a_{k}} \int_{-\infty}^{\infty} h_{i}\left(\tau_{1}\right) \mathrm{e}^{\mathrm{j} \omega \tau_{1}} \mathrm{~d} \tau_{1} \times \int_{-\infty}^{\infty} h_{k}\left(\tau_{2}\right) \mathrm{e}^{-\mathrm{j} \omega \tau_{2}} \mathrm{~d} \tau_{2} \\
& \quad \times \frac{1}{2 \pi} \int_{-\infty}^{\infty} R_{f}(\lambda) \mathrm{e}^{-\mathrm{j} \omega \lambda} \mathrm{d} \lambda,
\end{aligned}
$$

where the substitution $\lambda=\tau+\tau_{1}-\tau_{2}$ was made. Next notice that

$$
\frac{1}{2 \pi} \int_{-\infty}^{\infty} R_{f}(\lambda) \mathrm{e}^{-\mathrm{j} \omega \lambda} \mathrm{d} \lambda=\frac{1}{2 \pi} \int_{-\infty}^{\infty} R_{f_{0}} \delta(\lambda) \mathrm{e}^{-\mathrm{j} \omega \lambda} \mathrm{d} \lambda=\frac{R_{f_{0}}}{2 \pi} \triangleq S_{f_{0}},
$$

where the constant $S_{f_{0}}$ represents the spectrum of the uncorrelated stochastic excitation. Also, by using equations (5) and (6),

$$
\begin{aligned}
& \int_{-\infty}^{\infty} h_{i}\left(\tau_{1}\right) \mathrm{e}^{-\mathrm{j} \omega \tau_{1}} \mathrm{~d} \tau_{1}=\frac{1}{s_{i}-\mathrm{j} \omega} \triangleq H_{i}(\mathrm{j} \omega), \\
& \int_{-\infty}^{\infty} h_{i}\left(\tau_{1}\right) \mathrm{e}^{\mathrm{j} \omega \tau_{1}} \mathrm{~d} \tau_{1}=\frac{1}{s_{i}+\mathrm{j} \omega} \triangleq \bar{H}_{i}(\mathrm{j} \omega), \\
& \int_{-\infty}^{\infty} h_{i}^{*}\left(\tau_{1}\right) \mathrm{e}^{-\mathrm{j} \omega \tau_{1}} \mathrm{~d} \tau_{1}=\frac{1}{s_{i}^{*}-\mathrm{j} \omega} \triangleq \bar{H}_{i}^{*}(\mathrm{j} \omega), \\
& \int_{-\infty}^{\infty} h_{i}^{*}\left(\tau_{1}\right) \mathrm{e}^{\mathrm{j} \omega \tau_{1}} \mathrm{~d} \tau_{1}=\frac{1}{s_{i}^{*}+\mathrm{j} \omega} \triangleq H_{i}^{*}(\mathrm{j} \omega),
\end{aligned}
$$

based on which [as well as equations (53) and (54)] $S_{k}(\omega)$ may be finally expressed as

$$
\begin{aligned}
S_{k}(\omega)= & \operatorname{Re}\left[F_{k}(\mathrm{j} \omega)\right] \\
= & \operatorname{Re}\left[S _ { f _ { 0 } } \sum _ { i = 1 } ^ { n } \left[\frac{\phi_{m i} \phi_{l i} \phi_{m k} \phi_{l k}}{a_{i} a_{k}} \bar{H}_{i}(\mathrm{j} \omega) H_{k}(\mathrm{j} \omega)+\frac{\phi_{m i} \phi_{l i} \phi_{m k}^{*} \phi_{l k}^{*}}{a_{i} a_{k}^{*}} \bar{H}_{i}(\mathrm{j} \omega) \bar{H}_{k}^{*}(\mathrm{j} \omega)\right.\right. \\
& \left.\left.+\frac{\phi_{m i}^{*} \phi_{l i}^{*} \phi_{m k} \phi_{l k}}{a_{i}^{*} a_{k}} H_{i}^{*}(\mathrm{j} \omega) H_{k}(\mathrm{j} \omega)+\frac{\phi_{m i}^{*} \phi_{l i}^{*} \phi_{m k}^{*} \phi_{l k}^{*}}{a_{i}^{*} a_{k}^{*}} H_{i}^{*}(\mathrm{j} \omega) \bar{H}_{k}^{*}(\mathrm{j} \omega)\right]\right]
\end{aligned}
$$

Remark. In the special case of proportionally damped systems, the above expression for $S_{k}(\omega)$ reduces to

$$
S_{k}(\omega)=\operatorname{Re}\left[F_{k}(\mathrm{j} \omega)\right]=S_{f_{0}} \sum_{i=1}^{n} \phi_{m i} \phi_{l i} \phi_{m k} \phi_{l k} \times \operatorname{Re}\left[G_{i}^{*}(\mathrm{j} \omega) G_{k}(\mathrm{j} \omega)\right],
$$

with

$$
G_{i}(\mathrm{j} \omega) \triangleq-\left(\frac{H_{i}(\mathrm{j} \omega)}{a_{i}}+\frac{\bar{H}_{i}^{*}(\mathrm{j} \omega)}{a_{i}^{*}}\right)=\frac{1}{\omega_{n_{i}}^{2}-\omega^{2}+\mathrm{j} 2 \zeta_{i} \omega_{n_{i}} \omega}
$$

representing the complex frequency response function for the $i$ th normal co-ordinate system [25]. 
Example (continued). Consider again the two-degree-of-freedom proportionally damped system of Figure 1, and more specifically the point receptance $v_{1} / f_{1}$ and transfer receptance $v_{2} / f_{1}$ transfer functions. The point receptance case is examined in Figures 4(a) and $4(\mathrm{~b})$, where the decomposition of the impulse response $x(t)$ in terms of the modal impulse responses $x_{1}(t)$ and $x_{2}(t)$ is shown, along with the decomposition of the vibration response spectrum $S_{v_{1}}(\omega)$ in terms of $S_{1}(\omega)$ and $S_{2}(\omega)$. As it may be readily observed, both modal impulse responses are positively correlated with $x(t)$, and are therefore characterized by positive dispersions (a result which is in agreement with the dispersion analysis

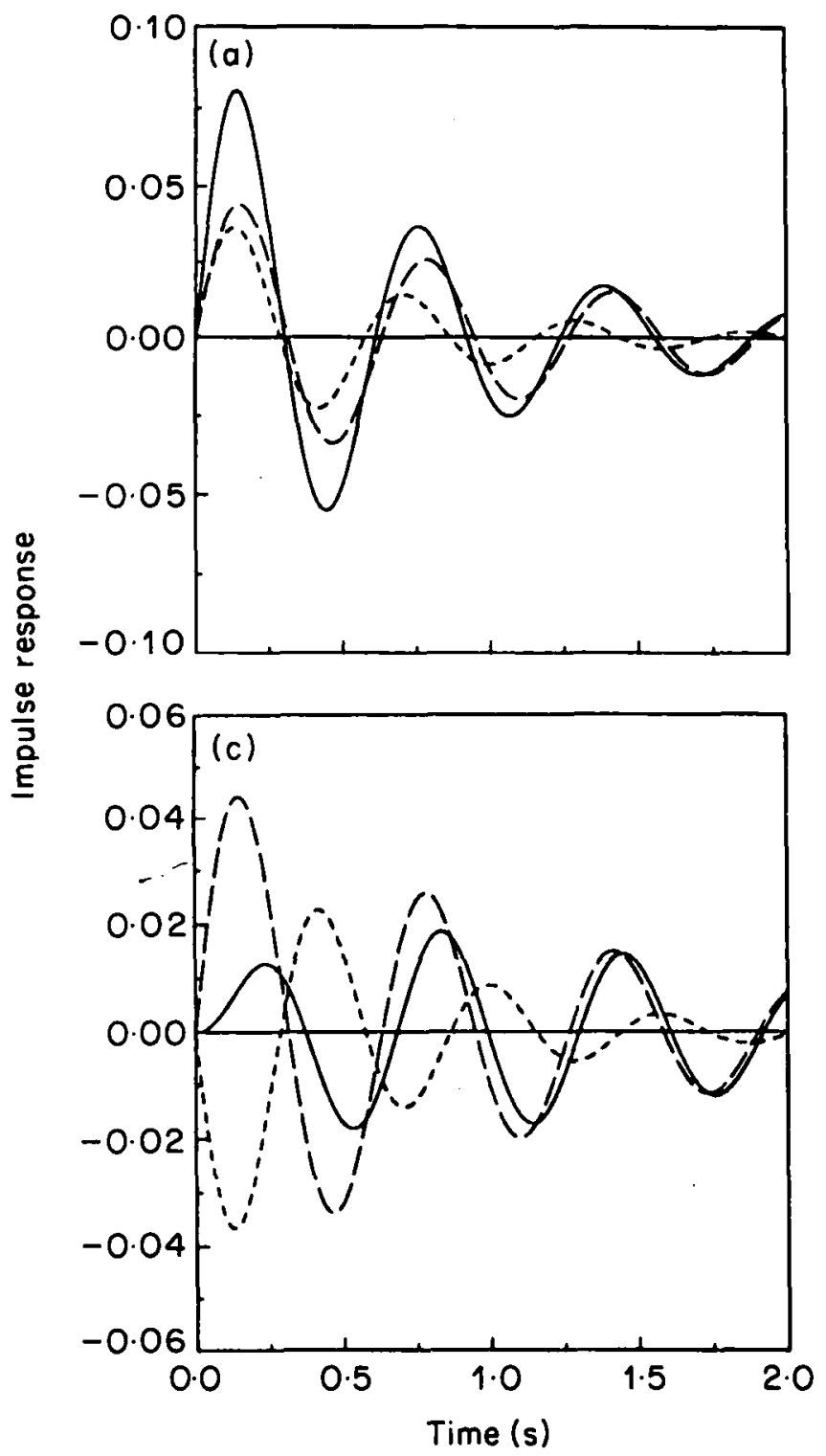

Figure 4. (a) The first $\left[x_{1}(t)\right],(--)$ second $\left[x_{2}(t)\right],(--)$ and total $[x(t)],(-)$ impulse response functions of the transfer function $v_{1} / f_{1}$. (b) The decomposition of the vibration signal spectrum $S_{v 1}(\omega)$, $(-)$ into the modal components $S_{1}(\omega),(--)$ and $S_{2}(\omega),(--)$ for the transfer function $v_{1} / f_{1}$. (c) The first [ $x_{1}(t)$ ], $(--)$ second $\left[x_{2}(t)\right],(---)$ and total $[x(t)],(-)$ impulse response functions of the transfer function $v_{2} / f_{1}$. (d) The decomposition of the vibration signal spectrum $S_{v 2}(\omega),(-)$ into the modal components $S_{1}(\omega)$, $(--)$ and $S_{2}(\omega),(---)$ for the transfer function $v_{2} / f_{1}$. Two-degree-of-freedom system. 


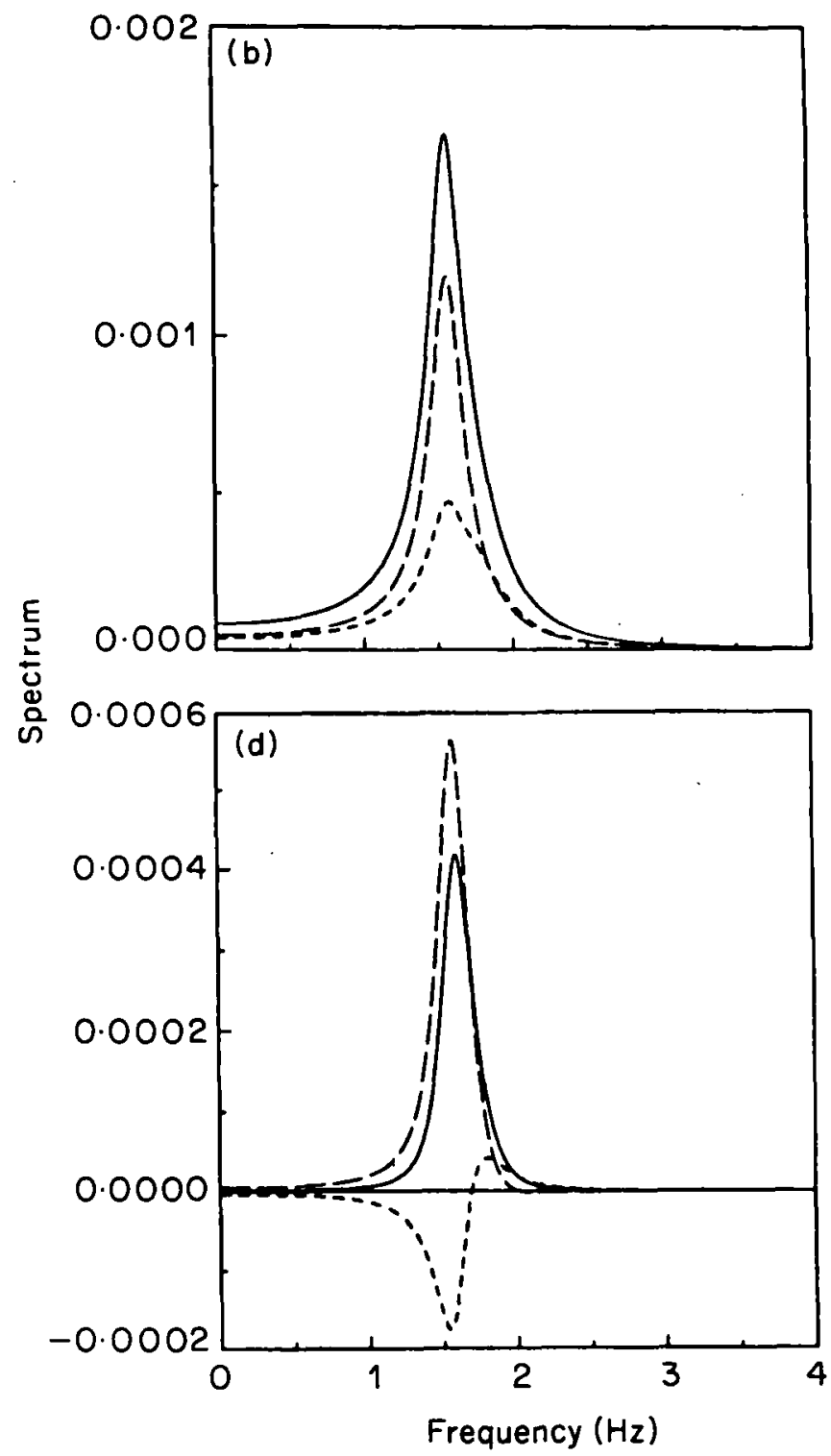

Figure 4-continued.

results for this system and also Corollary 2). The same conclusion may be reached by examining the spectral results, from which it is evident that both modes contribute positively to the vibration signal spectrum (in this case, in particular, they contribute positively at each and every frequency $\omega$ ) and therefore add to the vibration signal energy. The particular contribution of each mode to the vibration energy can be computed as the area between the corresponding $S_{k}(\omega)$ and the frequency axis.

In the transfer receptance case, however, the modal impulse response $x_{2}(t)$ is negatively correlated with the overall impulse response $x(t)$, as indicated in Figure 4(c), and the second mode is therefore characterized by negative dispersion. This is in agreement with the dispersion analysis results of the system (see the discussion in the previous subsection) and also the spectral results, as it is evident that $S_{2}(\omega)$ has a negative overall contribution 
to the vibration signal spectrum $S(\omega)$ (although for a particular frequency range the contribution of the second mode to the spectrum is in fact positive) (Figure 4(d)).

\section{CONCLUSIONS}

In this paper, the problem of quantitatively assessing the relative importance of a structural system's vibrational modes was addressed, and an appropriate and novel dispersion analysis methodology was introduced. The proposed methodology assesses the significance of each mode in an objective and physically meaningful way by determining the mode's contribution (modal dispersion) to the total vibration signal energy under broadband stochastic excitation conditions while fully accounting for all cross-correlation (interaction) effects among the various modes.

Physical interpretations of the modal dispersion have been derived in both the correlation and spectral domains based on the notion of modal response functions, and the dispersion of a mode has been shown to be equal to the deterministic (stochastic) cross-correlation between the corresponding modal and the total vibration responses due to a deterministic delta function (uncorrelated stochastic signal). The modal dispersion may be thus clearly interpreted as a measure of the mode's influence in shaping the total vibration response.

Explicit and physically significant expressions that relate the modal dispersion to the structure's global and local characteristics have been derived, and the phenomenon of modes characterized by negative dispersions investigated. Conditions for the existence of such modes were derived, and physical interpretations provided. Negative dispersion modes were thus shown to be encountered in cases of strong modal interference, specifically in cases where the modal cross-correlation effects dominate over those of the modal autocorrelation; something that can, for instance, happen in cases of structural systems characterized by closely spaced modes. Once encountered, negative dispersion modes have a negative overall contribution to the vibration response power spectrum, and act in a way that tends to suppress the structural system's total response.

In the second part of the paper [23], the problem of stochastic MIMO experimental modal analysis will be considered, and a realistic and effective approach will be developed that uses the dispersion analysis methodology for model analysis and reduction/distinction between structural and extraneous modes.

\section{REFERENCES}

1. S. R. Ibrahim and E. C. Mikulcik 1973 Shock and Vibration Bulletin 43, 21-37. A time domain vibration test technique.

2. D. L. Brown, R. J. Allemang, R. Zimmerman and M. Mergeay 1979 SAE Transactions 88, 828-846. Parameter estimation techniques for modal analysis.

3. H. Vold and T. RocKLIN 1982 Proceedings of the 1 st International Modal Analysis Conference, Orlando, Florida, 542-548. The numerical implementation of a multi-input modal estimation method for mini-computers.

4. J. N. JUANG and R. S. PAPPA 1985 American Institute of Aeronautics and Astronautics Journal of Guidance, Control, and Dynamics 8, 620-627. An eigensystem realization algorithm for modal parameter identification and model reduction.

5. J. M. Leuridan, D. L. Brown and R. J. Allemang 1986 Journal of Vibration, Acoustics, Stress, and Reliability in Design 108, 1-8. Time domain parameter identification methods for linear modal analysis: a unifying approach.

6. H. Kano 1989 Journal of Dynamic Systems, Measurement, and Control 111, 146-152. An identjfication method of multiinput multioutput linear dynamic systems for the experimental modal analysis of mechanical structures.

7. C. P. FRITZEN 1986 Journal of Vibration, Acoustics, Stress, and Reliability in Design 108, 9-16. Identification of mass, damping, and stiffness matrices of mechanical systems. 
8. R. BEN MRAD and S. D. FASSors 1991 Journal of Vibration and Acoustics 113, 354-361. Recursive identification of vibrating structures from noise-corrupted observations, part I : identification approaches.

9. R. BEN MRAD and S. D. FAssors 1991 Journal of Vibration and Acoustics 113, 362-368. Recursive identification of vibrating structures from noise-corrupted observations, part II : performance evaluation via numerical and laboratory experiments.

10. J. E. LEE and S. D. FAssois 1990 Proceedings of the 8 th International Modal Analysis Conference, Kissimmee, Florida 2, 1424-1433. A stochastic suboptimum maximum likelihood approach to structural dynamics identification.

11. K. J. KIM, K. F. Eman and S. M. Wu 1984 International Journal of Machine Tool Design and Research 24, 161-169. Identification of machine tool structures by the dynamic data system approach.

12. P. Davies and J. K. Hammond 1984 Journal of Vibration, Acoustics, Stress, and Reliability in Design 106, 40-48. A comparison of Fourier and parametric methods for structural system identification.

13. S. M. Pandit and N. P. Merta 1988 Journal of Sound and Vibration 122, 413-422. Data dependent systems approach to modal analysis, part I: theory.

14. S. M. Pandit and N. P. Mehta 1988 Journal of Sound and Vibration 122, 423-432. Data dependent systems approach to modal analysis, part II : application to structural modification of a disc-brake rotor.

15. Y. C. Shin, K. F. EMAN and S. M. WU 1989 Journal of Engineering for Industry 111, 116-124. Experimental complex modal analysis of machine tool structures.

16. S. D. FAssois, K. F. EMAN and S. M. WU 1990 Journal of Vibration and Acoustics 112, 98106. A linear time-domain method for structural dynamics identification.

17. S. Hu, Y. B. CHEN and S. M. WU 1989 Proceedings of the 12 th Biennial Conference on Mechanical Vibration and Noise, Montreal, Quebec, Canada, 259-265. Multi-output modal parameter identification by vector time series modeling.

18. J. E. LEE and S. D. FAssors 1992 Journal of Vibration and Acoustics 114, 260-271. Suboptimum maximum likelihood estimation of structural parameters from multiple-excitation vibration data.

19. S. M. KAY and S. L. MARPLE 1981 Proceedings of the Institute of Electrical and Electronic Engineers 69, 1380-1419.

20. D. Bonnecase, M. Prevosto and A. Benveniste 1990 Proceedings of the 8th International Modal Analysis Conference, Kissimmee, Florida 1, 382-388. Application of a multidimensional ARMA model to modal analysis under natural excitation.

21. M. Gevers and V. Wertz 1987 Control and Dynamics Systems: Advances in Theory and Applications (C. T. Leondes, editor) 26, 35-86. Academic Press. Techniques for the selection of identifiable parametrizations for multivariable linear systems.

22. R. H. JoNEs 1985 Time Series Analysis of Irregularly Observed Data (E. Parzen, Editor) Notes in Statistics 25, 158-188. Springer-Verlag. Fitting multivariable models to unequally spaced data.

23. S. D. FASSOIS and J. E. LEE 1992 Journal of Sound and Vibration 161, 57-87. On the problem of stochastic experimental modal analysis based on multiple-excitation multiple-response data, part II : the modal analysis approach.

24. S. M. Pandit and S. M. WU 1983 Time Series and System Analysis with Applications. New York: John Wiley.

25. L. Meirovitch 1967 Analyfical Methods in Vibrations. New York: Macmillan.

26. I. Elishakoff 1983 Probabilistic Methods in the Theory of Structures. New York: John Wiley.

27. S. I. Baskakov 1986 Signals and Circuits. Moscow: MIR.

\section{APPENDIX: NOMENCLATURE}

C

$D_{k}(\tau)$

$D_{k}(0)$

$\mathbf{f}(\mathrm{t})$

$F_{k}(\mathrm{j} \omega)$

$G_{i}(j \omega)$

$\mathbf{j}$

viscous damping matrix

part of $R_{v_{m}}(\tau)$ associated with the $k$ th mode

$k$ th modal dispersion

(vector) force excitation

Fourier transform $D_{k}(\tau)$

frequency response on the $i$ th normal co-ordinate system

imaginary unit (if not an index)

stiffness matrix 
$\mathbf{M}$

$n$

$x(t)$

$x_{i}(t)$

$R_{f}(\tau)$

$R_{f 0}$

$R_{v_{m}}(\tau)$

$s_{i}$

$S_{f 0}$

$S_{k}(\omega)$

$S_{v_{m}}(\omega)$

$\mathbf{v}(t)$

$v_{m}(t)$

$v_{m_{k}}(t)$

$\beta_{k k}$

$\beta_{k}$

$\delta_{k}$

$\Delta$

$\varepsilon_{\boldsymbol{\omega}_{i k}}$

$\zeta_{k}$

$\omega_{n k}$

$\omega_{d_{k}}$

$\phi_{k}$

$\phi_{i k}$

\section{Conventions}

$\mathrm{E}\{\cdot\}$

$\operatorname{Re}(\cdot)$

$x_{i}$

$\mathbf{x}(t)$

$\{\mathbf{x}(t)\}$

$T$

capital bold face lower-case bold face mass matrix

number of degrees of freedom

impulse response of a scalar receptance transfer function

$i$ th modal impulse response function (part of $x(t)$ )

autocorrelation of the excitation $\left\{f_{l}(t)\right\}$

intensity of an uncorrelated force excitation signal

autocovariance of the $m$ th vibration displacement signal

system eigenvalue

uncorrelated stochastic force excitation spectral intensity

$k$ th mode spectral contribution (real part of $F_{k}(\mathrm{j} \omega)$ )

spectrum of $\left\{v_{m}(t)\right\}$

(vector) vibration displacement

the $m$ th component of the vibration displacement vector

$k$ th modal response due to uncorrelated force excitation (part of $v_{m}(t)$ )

$k$ th modal autocorrelation

$k$ th modal cross-correlation

$k$ th mode dispersion percentage (normalized dispersion)

$=1-\varepsilon_{\omega_{12}}$

$=\omega_{n_{i}} / \omega_{n_{k}}$, natural frequency ratio

$k$ th damping factor

$k$ th natural frequency

$k$ th damped natural frequency

$k$ th mode shape

$i$ th element of $k$ th mode shape

denotes statistical expectation

denotes real part

$i$ th scalar component of vector $\mathbf{x}$

value at time $t$ of the analog signal $x$

signal $x$

(superscript) denotes complex conjugate

(superscript) denotes transpose

denotes matrix quantity

denotes (column) vector quantity

autoregressive moving-average

eigensystem realization algorithm

frequency domain method

multiple-input multiple-output

noise-to-signal 